\title{
CircRNA CircCCT3 Acts as a Sponge of miR-613 to Promote Tumor Growth of Pancreatic Cancer Through Regulating VEGFA/VEGFR2 Signaling
}

\section{Ji-Ping Hou}

Tianjin Baodi Hospital

\section{Xue-Bo Men}

Tianjin Baodi Hospital

\section{Lian-Ying Yang}

Tianjin Baodi Hospital

\section{En-Kun Han}

Tianjin Baodi Hospital

\section{Chun-Qi Han}

Tianjin Baodi Hospital

\section{Li-Bin Liu ( $\sim$ baodillb@163.com )}

Tianjin Baodi Hospital

\section{Research}

Keywords: CircCCT3, Pancreatic cancer, miR-613, VEGFA/VEGFR2 signaling

Posted Date: March 4th, 2021

DOl: https://doi.org/10.21203/rs.3.rs-268983/v1

License: (1) This work is licensed under a Creative Commons Attribution 4.0 International License.

Read Full License

Version of Record: A version of this preprint was published at Balkan Medical Journal on July 6th, 2021. See the published version at https://doi.org/10.5152/balkanmedj.2021.21145. 


\section{Abstract}

Objective This study was aimed at investigating the involvement of CircССТ3 in PC and study its interactions and functioning during the PC progression in vitro and in vivo by the use of molecular biology and bioinformatic methods.

Methods The expressions of CircCCT3 and miR-613 in pancreatic carcinoma tissues and cell lines were evaluated by quantitative realtime PCR . The relationship between clinical pathologic features as well as survival rate and CircCCT3 expression was analyzed with Chi-square test and Kaplan-Meier method. CCK-8, wound healing , transwell assays and FITC-AnnexinV/PI assay were used to assess cell proliferation, migration, invasion and apoptosis after CircCCT3 overexpression or downregulation. DualLuciferase reporterassay, RNA immunoprecipitation (RIP) ,RNA pull down and fluorescence in situ hybridization(FISH) assays were performed to validate the potential interaction of CircCCT3, miR-613 and VEGFA.Nude mouse xenograft tumor assay was used to detect CircCCT3 effects on pancreatic tumorigenesis in vivo.Western blotting analysis was performed to examine the VEGFA and VEGFR2 protein expressions following.

Results CircCCT3 expression was significantly increased in PC tissues and cell lines. CircCCT3 expression was negatively correlated with miR-613 expression. Moreover, it was found that CircCCT3 promote cell proliferation, migration, invasion and inhibited cell apoptosis in PC cells. CircCCT3 acted as a sponge for miR-613 to facilitate VEGFA and VEGFR2 expression. si-CirCCT3also inhibited tumor growth of PC in nude mice.si-CircCCT3 reduced VEGFA and VEGFR2 expression, whereas overexpression of CircCCT3 increased VEGFA and VEGFR2 expression.

Conclusions Increased CircCCT3 suggests a poor prognosis in PC patients and promotes the migration and invasion through targeting VEGFA/VEGFR2 signaling. CirCCCT3 may serve as a potential and promising therapeutic target for $\mathrm{PC}$ treatment.

\section{Introduction}

Pancreatic cancer $(\mathrm{PC})$ is one of the most lethal malignancies and aggressive gastrointestinal tumors, remaining the lowest 5 -year relative survival rate of $9 \%{ }^{[1]}$. Since its aggressive nature, late diagnosis and limitations of existing chemo and radiation therapies, PC has become the fourth leading cause of cancer types for the estimated death and has been predicted to become the second cause of cancer-related deaths in developed countries by $2030^{[2]}$. Therefore, understanding the potential mechanism that promotes PC progression is vital for developing effective diagnostic techniques and therapeutic strategies.

Circular RNAs (circRNAs) are defined as closed and single-stranded RNAs derived from back-spliced premRNA ${ }^{[3]}$. Initially, circRNAs are discovered 20 years ago and believed as by-products of aberrant splicing [4]. Recent studies have shown that circRNAs play various effects on normal metabolism and 
pathophysiological process ${ }^{[5]}$. Accruing evidences have shown that circRNAs function as modulators in cancer development, and they may be novel biomarkers and therapeutic targets ${ }^{[6]}$. CircCCT3 (circBase ID: hsa_circ_0004680) is originated from exons $3-5$ and introns $3-4$ of chaperonin containing TCP1 subunit 3 (ССТ3) by back-splicing ${ }^{[7]}$, and is identified to be involved in the progression of hepatocellular carcinoma $^{[7]}$ and colorectal cancer ${ }^{[8]}$.So far, the role of CircCCT3 on PC progression has not been investigated before.

miRNAs are short non-coding RNAs that contains about 22 nucleotides ${ }^{[9]}$. Emerging evidence has been demonstrated that miRNAs are involved in cancer initiation and progression ${ }^{[9]}$. So, miRNAs could be oncogenes or tumor suppressor genes mainly by regulating targeting genes that contribute to cellular processes such as cell cycle, mobility and survival ${ }^{[10]}$. miR-613 has been demonstrated to be downregulated in several types of cancers including renal cell carcinoma, glioma, breast cancer, and cervical cancer,implying that miR-613 may act as a tumor suppressor gene ${ }^{[11-14]}$. However, its function and mechanism in $\mathrm{PC}$ remain unclear.

Vascular endothelial growth factor A (VEGFA) is one member of the VEGF family proteins ${ }^{[15] . V E G F A ~ i s ~}$ regarded as one of the most important in malignant diseases since it is the most predominant isoform secreted by human tumors ${ }^{[16]}$ including $\mathrm{PC}^{[17,18]}$.Also the VEGFA/VEGFR2 signaling has been implicated in different cancer progression $[19,20]$.

Therefore, the scope of our study was performed to analyze the expression pattern of CircCCT3 in PC ,explore the correlation of CircCCT3 with PC phenotype and investigate whether CircCCT3 leads to PC progression through targeting miR-613 and regulating VEGFA/VEGFR2.

\section{Materials And Methods}

\section{Tissue Specimens and Cell Culture}

30 paired PC tissues and non-tumor adjacent tissues were collected from the Tianjin Baodi Hospital . Inclusion criteria: (1) there was no adjuvant therapy before surgery, including chemotherapy and radiotherapy. (2)PC pathology was confirmed by two experienced pathologists after surgery.(4) The patient could tolerate the operation without evident heart, brain and lung diseases. Exclusion criteria: (1) preoperative chemotherapy, radiotherapy and other related adjuvant therapies. (2) Patients could not tolerate surgery. This investigation was approved by the Ethics Committee of Tianjin Baodi Hospital. All patients agreed the protocol and signed the informed consent. This investigation was conducted in accordance with the Declaration of Helsinki.

Human pancreatic duct epithelial cell (H6c7) and human PC cell lines (Patu8988, SW1990, BxPC-3 and Panc02) were obtained from ATCC (Manassas, VA, USA). All cells were cultured in Roswell Park Memorial Institute-1640 (RPMI-1640) medium (Invitrogen, Carlsbad, CA, USA) containing 10\% fetal bovine serum (FBS; Gibco, Rockville,MD, USA) and incubated in a humidified atmosphere with $5 \% \mathrm{CO}_{2}$ at $37^{\circ} \mathrm{C}$. 


\section{Quantitative Real Time Polymerase Chain Reaction (qRT-PCR)}

Total RNA was extracted by TRIzol (Invitrogen,Carlsbad, CA, USA) reagent and cDNA was synthesized using reverse transcription reaction kit (TaKaRa, Komatsu, Japan) following the instruction. qRT-PCR was performed by SYBR Premix DimerEraser (TaKaRa, Komatsu, Japan) on a LightCycler 480 PCR System (Roche, Rotkreuz,Switzerland). Relative expression of RNAs was calculated by $2^{-\Delta \Delta C T}$ method and normalized to $\mathrm{U} 6$ or glyceraldehyde phosphate dehydrogenase (GAPDH). Primers are listed as below:CircCCT3 Forward primer: GGACCCAGGATGAAGAGGTT;CircCCT3 Reverse primer:

CATTGGGTCCAAAAGCATCT; miR-613 Forward primer: GTGAGTGCGTTTCCAAGTGT; miR-613 Reverse primer: GGGTCCCTTCACACTTGGAA ; GAPDH: Forward primer: 5'-ACGCTGCATGTGTCCTTAG-3'; Reverse primer: 5'-GAGCCTCTTATAGCTGTTTG-3'; U6: Forward primer: 5'-CTCGCTTCGGCAGCACA-3'; Reverse primer: 5'-AACGCTTCACGAATTTGCGT-3'.

\section{CCK-8 assay}

$2 \times 10^{5}$ cells in each well were inoculated in $96-$ well plates. Medium was used as blank control. At posthypoxiareoxygenation, indicated cells in per well were added $10 \mu \mathrm{L}$ of CCK-8 (Beyotime Institute of Biotechnology, Beijing, China) solution, and kept at $37^{\circ} \mathrm{C}$ for $2 \mathrm{~h}$. The OD value of $450 \mathrm{~nm}$ was detected. The final data was represented as cell viability.

\section{Apoptosis assay}

For the apoptosis analysis, $1 \times 10^{6}$ cells were seeded in a $60 \mathrm{~mm}$ plate, treated with OE-CircCCT3 or CCT3 siRNA, and cultured for $24 \mathrm{~h}$. After harvesting, $1 \times 10^{5}$ cells were suspended in a $1 \mathrm{x}$ binding buffer provided with the Annexin V-FITC Apoptosis Detection kit II (BD Bioscience, San Jose, CA, USA), then stained with FITC Annexin V(BD Bioscience) and PI (Sigma-Aldrich, St. Louis, MO, USA). Fluorescence was detected with a BD Accuri $\mathrm{C} 6$ flow cytometer (BD Bioscience), and the data were analyzed with the BD Accuri C6 software (BD Bioscience).

\section{Wound-Healing Assay}

Cells were plated into six-well plates and grown to nearly $90 \%$ confluence. The same size scratch was made through the cell monolayer using a $200 \mu \mathrm{L}$ disposable pipette tip. After washing with PBS, fresh culture medium was added, and the cells were incubated at $37^{\circ} \mathrm{C}$ under an atmosphere with $5 \% \mathrm{CO}_{2}$. Wound closure was imaged at 0 and $48 \mathrm{~h}$.

\section{Bioinformatic Prediction}

Circular RNA Interactome software (https://circinterac tome.nia.nih.gov) was used to predict CircCCT3miRNA interactions, while the mRNA targets of miR-613 were predicted by TargetScan software (http://www.targetscan.org)

\section{Luciferase Reporter Assay}


First, a luciferase reporter vector (pGL3-Firefly_Luciferase-Renilla-Luciferase) with VEGFA 3' UTR or CircCCT3 was built, and the mutant vectors were constructed by GeneChem (Shanghai, PRChina). A luciferase vector and miR-613 mimic or mimic negative control were cotransfected into cells and incubated for $24 \mathrm{~h}$. A dual luciferase reporter assay detection kit (Promega, WI, USA) was used to detect firefly and Renilla luciferase activities, which were measured on a Fluoroskan Ascent device (Thermo Fisher Scientific, USA).

\section{Fluorescence in situ hybridization}

Cy3-CircCCT3 and fluorescein isothiocyanate (FITC)-miR-613 probes were synthesized and obtained from RiboBio (Guangzhou,PR China). Hybridization assays were performed using a FISH Detection Kit (RiboBio, PR China). All images were captured by a confocal microscope (FV1000; Olympus, Tokyo, Japan).

\section{RNA Immunoprecipitation (RIP) Assay}

A RIP kit (Millipore, Billerica, MA, USA)was purchased for performing the RIP assay. PC cells were collected, and lysed by RIP lysis buffer. Then, the cell lysates were incubated with anti-Argonaute2 (Ago2) or immunoglobulin G (IgG; negative control) antibody in RIP buffer at $4^{\circ} \mathrm{C}$ overnight. Co-precipitated RNA was isolate dafter digestion with $150 \mu \mathrm{L}$ protease $\mathrm{K}$. The expression of CircCCT3 and miR-613 was detected.

\section{Pulldown assay with biotinylated CircCCT3 and miR-613}

Probe Panc02 cells $\left(1 \times 10^{7}\right)$ were obtained, lysed, and sonicated as indicated. To generate the probecoated beads, C-1 magnetic beads (Life Technologies) were coincubated with the CircCCT3 probe for 2.5 $h$ at $25^{\circ} \mathrm{C}$. Then, the CircCCT3 probe or oligo probe was coincubated with cell lysates at $4^{\circ} \mathrm{C}$ overnight. RNA was eluted and extracted by wash buffer and used for qRT-PCR. The CircCCT3 probe with biotinylation was synthesized and purchased from RiboBio (Guangzhou, PR China). The miR-613 was also tested using the same procedure.

\section{Western Blotting}

Cells were lysed with precooled radio-immunoprecipitation assay lysis buffer supplemented with protease inhibitor (Beyotime Institute of Biotechnology, Shanghai, China) for 30 min on ice. The supernatant was collected after centrifugation at $14,000 \mathrm{rpm}$ at $4{ }^{\circ} \mathrm{C}$ for $20 \mathrm{~min}$. The protein concentration was determined using a bicinchoninic acid (BCA) protein concentration determination kit (RTP7102; Real-Times Biotechnology Co.,Ltd., Beijing, China). The samples $(20 \mu \mathrm{g})$ were subjected to $10 \%$ sodium dodecyl sulfate-polyacrylamide gel electrophoresis and then transferred to polyvinylidene difuoride membranes. A GAPDH antibody was used as an internal reference. The membranes were washed with TBST and incubated with goat anti-mouse/rabbit antibody. Color development was performed using the chemiluminescence detection method, and images of protein bands were obtained for analyses. The 
primary and secondary antibodies were as follows: anti-GAPDH monoclonal antibody (Cell Signaling Technology, Danvers, MA,USA; 5174S, diluted 1/1000), anti-VEGFA (Abcam, Cambridge,UK;ab214424 diluted 1/1000), anti-VEGFR2 (Abcam,Cambridge,UK; ab134191, diluted 1/1000), goat anti-mouse immunoglobulin G (IgG) (ProteinTech; SA00001-1, diluted 1/3000), and goat anti-rabbit IgG (ProteinTech; SA00001-2, diluted 1/3000). Each western blotting was repeated at least three times.

\section{Xenograft Tumor Model}

Animal experiments were conducted with the permission of the animal care and use committee of Baodi Clinical College of Tianjin Medical University and performed in accordance with the guidelines of the National Animal Care and Ethics Institution. Panc02 cell line stably transfected with sh-CircCCT3 or sh-NC was established. Afterwards, Panc02 cells stably transfected with sh-CircCCT3 or sh-NC were subcutaneously inoculated into the right flank of BALB/c male nude mice (Model Animal Research Center Of Nanjing University, Nanjing, China) in sh-NC group and sh-CircCCT3 group. The volume of tumors was recorded every week. After inoculation for 28 days, these nude mice were euthanized and the tumors were dissected and weighed. The expression of CircCCT3 was detected by qRT-PCR.

\section{Statistical Analysis}

All data were described as mean \pm SD and analyzed with GraphPad Prism 7.0 software (GraphPad Prism, San Diego, CA, USA). One-way ANOVA with a Bonferroni correction or Student's t-test was used to analyze the differences between groups. The association between CircССТ3 expression and clinicopathological features was evaluated using Chi-square test. Survival data were analyzed using Kaplan-Meier method and the log-rank test. The correlation of CircCCT3 expression between miR-613 expression was carried out using Spearman's method. $P<0.05$ was regarded as statistical significance.

\section{Results}

\section{CircCCT3 is increased in clinical PC tissues}

To investigate whether CircCCT3 played a role on PC metastasis and progression, we tested the expression level of CircCCT3 in PC and non-PC tissue. We observed that CircCCT3 levels in human PC tumors were significantly higher compared with adjacent non-PC tissues $(P<0.001)$ (Fig. 1A), whereas miR-613 expression in PC tissue was markedly decreased compared to adjacent non-PC tissue. Higher levels of CircCCT3 in PC patients correlated with vascular invasion $(P=0.011)$, peritoneal metastasis $(P=$ $0.013)$,lymph node metastasis $(P=0.020)$ and clinical progression $(P=0.009)($ Table 1$)$. In addition,CircCCT3 expression was negatively correlated with miR-613 expression in PC tissue. PC patients with high CircCCT3 expression $(n=15)$ exhibited significantly poorer overall survival rate than those patients with low CircCCT3 expression $(n=15)$ as defined by log-rank test $(P=0.013)$

(Fig. 1D).These results implicated that CircCCT3 may serve as an oncogene in PC. 
Table 1

CircCCT3 Expression and its correlation with progression of PC

\begin{tabular}{|c|c|c|c|c|}
\hline \multirow[t]{2}{*}{ Characteristics } & \multirow[t]{2}{*}{ Case (No.) } & \multicolumn{2}{|c|}{ Expression of CircCCT3 } & \multirow[t]{2}{*}{$P$ value } \\
\hline & & High & Low & \\
\hline \multicolumn{5}{|l|}{ Age } \\
\hline$<50$ & 8 & 5 & 3 & 0.409 \\
\hline$\geq 50$ & 22 & 10 & 12 & \\
\hline \multicolumn{5}{|l|}{ Gender } \\
\hline Male & 16 & 9 & 7 & 0.538 \\
\hline Female & 14 & 6 & 8 & \\
\hline \multicolumn{5}{|l|}{ Location } \\
\hline Head & 11 & 6 & 5 & 0.705 \\
\hline Body/tail & 19 & 9 & 10 & \\
\hline \multicolumn{5}{|l|}{ Tumor Size(cm) } \\
\hline$<4$ & 20 & 12 & 8 & 0.245 \\
\hline$\geq 4$ & 10 & 3 & 7 & \\
\hline \multicolumn{5}{|c|}{ Tumor differentiation } \\
\hline High/medium & 12 & 7 & 5 & 0.176 \\
\hline Low & 18 & 8 & 10 & \\
\hline \multicolumn{5}{|c|}{ Vascular Invasion } \\
\hline Absent & 15 & 11 & 4 & $0.011^{*}$ \\
\hline Present & 15 & 4 & 11 & \\
\hline \multicolumn{5}{|l|}{ Peritoneal } \\
\hline \multicolumn{5}{|l|}{ Metastasis } \\
\hline No & 22 & 8 & 14 & $0.013^{*}$ \\
\hline Yes & 8 & 7 & 1 & \\
\hline \multicolumn{5}{|c|}{ Lymph Node Metastasis } \\
\hline Absent & 10 & 2 & 8 & $0.020^{\star}$ \\
\hline Present & 20 & 13 & 7 & \\
\hline
\end{tabular}




\begin{tabular}{|lclll|}
\hline Characteristics & Case (No.) & \multicolumn{2}{l|}{ Expression of CircCCT3 } & Pvalue \\
\cline { 1 - 3 } & & High & Low & \\
\hline Clinical Stage & & & & \\
\hline I/II & 13 & 3 & 10 & $0.009 * *$ \\
\hline III/IV & 17 & 12 & 5 & \\
\hline$* \star P<0.01, * P<0.05$ & & & & \\
\hline
\end{tabular}

\section{CircCCT3 expression was upregulated in PC cell line}

We next tested CircCCT3 level in different cell lines, we found CircCCT3 expressions in normal cell line H6c7 were significantly lower than PC tumor cell lines (Patu8988, SW1990, BxPC-3 and Panc02) ( $* \star P<$ 0.01)(Fig. 1A).To explore the effects of CircCCT3 on the biological function of Panc02 cells, we first explored cell proliferation via cell counting Kit-8 (CCK-8) assays and apoptosis through FITC-AnnexinV/PI assay. These results showed that overexpression of CircCCT3 increased cell proliferation of Panc02 cells compared with NC $(P<0.01)$, while silencing CircCCT3 suppressed cell proliferation $(P<0.05)$ (Fig. $2 \mathrm{~B})$. In contrast, the OE-CircCCT3 adding miR-613 mimic reversed the effect of the OE-CCT3 by decreasing cell growth (Fig. 2B).We also performed FITC-AnnexinV/PI assay to evaluate the potential function of CircCCT3 on cell apoptosis.We found cell apoptosis rate was significantly decreased in OE-CircCCT3 group $(P<0.05)$, while in si-CircCCT3 group the cell apoptosis rate was increased compared with NC group $(P<0.01)$. OE-CircCCT3 adding miR-613 had similar apoptosis rate in comparison with NC group

\section{CircССТ3 promotes the migration and invasion of $\mathrm{PC}$ both in vitro and in vivo}

In order to evaluate the potential role of CircCCT3 on the metastasis of PC, we introduced OE-CircCCT3 or CircCCT3 siRNA and CircCCT3 siRNA + miR-613 into Panc02 cells.

Subsequently, wound-healing assay was conducted, and data showed that overexpression of CircCCT3 significantly increased Panc02 cells migration in vitro (Fig. $2 A, B, P<0.05$ ). On the other hand, the migrated ability of Panc02 cells was significantly inhibited by CircCCT3 siRNA (Fig. 3A,B $P<0.05$ ). Cotransfection of OE-CircCCT3 and miR-613 mimic exhibited the similar effects compared with NC(Fig. 3A,B). In addition, percentages of invasion of CircCCT3 overexpressing Panc02 cells were significantly lower than those of NC cells, whereas si-CircCCT3 transfecting Panc02 had markedly decreased invasion numbers compared to NC cells (Fig. 3C,D). Co-transfection of OE-CircCCT3 and miR613 mimic exhibited the similar effects compared with NC (Fig. 3C,D). In short, these results demonstrated that CircCCT3 overexpression promotes the migratory and invasion capability of PC cells, and these effects could be reversed by adding miR-613 mimic. Last, in the xenograft tumor formation assay, we found sh-CirCCT3 inhibited tumor growth of PC compared with control at 4 weeks (Fig. 4A,B). The tumor volume and tumor weight in sh-CirCCT3 group were both significantly lower than sh-CircNC group (Fig. 4C,D). 


\section{CircCCT3 abolishes the interaction of miR-613 with the targets of VEGFA}

The predicted complementary binding sites at the 3'-UTR are shown in Fig. 5A. Bioinformatics analysis also showed that 3'-UTR of VEGFA were potential target genes of miR-613 (Fig. 5A). As shown next, the luciferase assay confirmed that CircCCT3 was also a target of miR-613 (Fig. 5C). Luciferase activity assay showed that miR-613 mimic led to a notable decrease in luciferase activity in CircCCT3-WT reporter compared with the mimic NC group, whereas miR-613 mimic had no obvious effect on luciferase activity in CircCCT3-MUT reporter(Fig. 5B). Similarly, miR-613 mimic contributed to the significantly reduction of luciferase activity in VEGFA-WT reporter in comparison with the mimic NC group,whereas miR-613 mimic had no obvious effect on luciferase activity in VEGFA-MUT reporter(Fig. 5D).In order to further identify the interaction among CircCCT3 and miR-613 in PC cells, we next carried out FISH, RNA pull-down and RIP assays. FISH technology demonstrated that CircCCT3 (red fluorescence) and miR-613 (green fluorescence) could be visualized in both Panc02 and BxPC3 cells, and colocalization was also observed (Fig. 6A).RIP assays disclosed that CircCCT3 and miR-613 expressions were substantially enriched by Ago2 antibody compared with control IgG antibody (Fig. 6B). The biotin-coupled probe pull-down assay was then performed and the results showed miR-613 was detected in the CircCCT3 pulled-down pellet compared with the control group(Fig. 6C). Also, CircCCT3 was detected in the miR-613 pulled-down pellet compared with the control group(Fig. 6D).

\section{CircCCT3 activated VEGFA/VEGFR2 signaling to facilitate PC progression}

VEGFA/VEGFR2 axis serves a crucial signaling in angiogenesis and metastasis of tumor progression [21]. To explore the role of CircCCT3 on the VEGFA/VEGFR2 axis, western blot analysis was performed, demonstrating that VEGFA and VEGFR2 expressions were both increased by transfecting OE-CircCCT3 compared with $\mathrm{NC}$, whereas these proteins were significantly downregulated by transfecting si-CircCCT3 in Panc02 cells(Fig. 7A-C). In addition, VEGFA and VEGFR2 expressions in co-transfection of OECircCCT3 and miR-613 mimic group were not significantly differed from NC groups. This finding suggested that CircCCT3 could regulate VEGFA/VEGFR2 axis in PC.

\section{Discussion}

The current study investigated the potential role of CircCCT3 in the PC development. We demonstrated for the first time that CircCCT3 was upregulated in PC and predicted poor prognosis in PC patients.

Functionally, we found that CircCCT3 knockdown markedly inhibited PC cell proliferation, migration, and invasion in vitro and decreased tumor growth in vivo. Through bioinformatics prediction, luciferase activity assay,RIP ,RNA pulldown and FISH, we discovered that miR-613 binds with CircCCT3 in PC cells. Finally, we identified CircCCT3 as an miR-613 sponge that upregulates the expression of VEGFA and VEGFR2 to promote PC progression.

Accumulating evidence suggests that CircRNAs may be used as tumor biomarkers and regulators because of their crucial involvement in various biological processes. Mechanistically, most identified circRNAs are mainly localized in the cytoplasm of the cell ${ }^{[22]}$,indicative of their roles in 
posttranscriptional regulation ${ }^{[23]}$. The ceRNA hypothesis indicates that circRNAs harbor MREs that bind miRNAs to reversely regulate the activity of the miRNAs ${ }^{[24]}$, thus attenuating the inhibitory effect on their target molecules. Mounting evidence has confirmed that some circRNAs can repress miRNA function and modulate target gene expression to play a tumor suppressor or oncogenic role by acting as miRNA sponges in different cancers including PC ${ }^{[25]}$.For example, CircRNA_000864 represses migration and invasion in PC Cells by targeting miR-361-3p ${ }^{[26]}$. Hsa_circ_0000069 knockdown could suppress tumorigenesis and malignant transformation via inhibition of STIL in PC ${ }^{27}$. hsa_circRNA_001587 upregulates SLC4A4 expression to inhibit migration, invasion, and angiogenesis of pancreatic cancer cells via binding to microRNA-223 ${ }^{[28]}$. circNFIB1 inhibits lymphangiogenesis and lymphatic metastasis via the miR-486-5p/PIK3R1/VEGF-C axis in pancreatic cancer ${ }^{[29]}$.

Angiogenesis is the formation of new vessels from existing capillaries, which is also an especially important process for tumor development and metastasis. Among many factors involved in the regulation of complex angiogenesis, VEGFA is a predominant stimulant ${ }^{[30]}$.

Continuous VEGF expression through VEGFR2 leads to the development and maintenance of a vascular network that promotes tumor growth and metastasis ${ }^{[31]}$. Drugs targeting the VEGF pathway have been studied as a single agent or in combination with chemotherapy to suppress the VEGF signaling pathway ${ }^{[32]}$.In our study, we found higher levels of CircССТ3 is correlated to vascular invasion, implicating CircCСТ3 is involved in the pathological process of angiogenesis in PC. We also discovered that overexpression of CircCCT3 increased VEGF and VEFGR2 protein expressions, suggesting CircCCT3 could promote VEGF/VEFGR2 signaling to facilitate angiogenesis in PC.

Our study has limitations. First, only 30 pairs of PC tissues were analyzed in this study due to limited number of available PC samples. Second, all the patient samples are collected from only one Chinese hospital. A larger number of PC samples should be tested at multiple centers and from other countries to further confirm the conclusions of this study. Third, we look forward to further research to explore why and how CircССТ3 was upregulated in PC, which will provide a deeper understanding of the molecular mechanism.

In conclusion, our study first revealed the novel function of CircCCT3 in PC progression. We illustrated that CircCCT3-mediated miR-613 and VEGFA/VEGFR2 signaling promote PC development and progression, providing a novel therapeutic target for PC therapy.

\section{Declarations}

\section{Acknowledgements}

All authors thank the patients donating their samples for this study

\section{Authors' contributions}


Ji-Ping Hou and Li-Bin Liu performed this study and drafted the article. Xue-Bo Men and Lian-Ying Yang carried out data statistics. En-Kun Han got the ethics approval. Chun-Qi Han helped edit the manuscript. The final version of the manuscript was approved by all authors.

\section{Funding}

No sources of funding were required for this work.

\section{Availability of data and materials}

All the data and materials are available.

\section{Ethics approval and consent to participate}

This investigation was approved by the Ethics Committee of Tianjin Baodi Hospital.

\section{Consent for publication}

All authors and studied participants agree to publish the paper.

\section{Competing interests}

The authors have no conflict of interest to declare.

\section{References}

1. Siegel RL, Miller KD, Jemal A. Cancer statistics, 2019. CA Cancer J Clin. 2019;691:7-34.

2. Rahib L, Smith BD, Aizenberg R, et al. Projecting cancer incidence and deaths to 2030: the unexpected burden of thyroid, liver, and pancreas cancers in the United States. Cancer Res 2014;7411:2913-21.

3. Chen L,Yang L.Regulation of circRNA biogenesis. RNA Biol. 2015; 12: 381-8.

4. Meng S, Zhou H, Feng Z, et al. CircRNA: functions and properties of a novel potential biomarker for cancer. Mol Cancer. 2017;16:94.

5. Rong $D$,Sun $H, L i Z$, et al. An emerging function of circRNA-miRNAs-mRNA axis in human diseases.[J] . Oncotarget. 2017; 8: 73271-73281.

6. Yin Y,Long J,He Q, et al. Emerging roles of circRNA in formation and progression of cancer.J Cancer. 2019;10: 5015-5021.

7. Lv B,Zhu W,Feng C.CircCCT3 Coptisine Blocks Secretion of Exosomal from Cancer-Associated Fibroblasts to Reprogram Glucose Metabolism in Hepatocellular Carcinoma.DNA Cell Biol. 2020, doi: 10.1089/dna.2020.6058. Online ahead of print.

8. Li W,Xu Y,Wang X, et al. CircCCT3 Modulates Vascular Endothelial Growth Factor A and Wnt Signaling to Enhance Colorectal Cancer Metastasis Through Sponging miR-613. DNA Cell Biol. 2020, 
39: 118-125.

9. Rupaimoole R, Slack FJ. MicroRNA therapeutics: towards a new era for the management of cancer and other diseases. Nat Rev Drug Dis. 2017;16:203-22.

10. Treiber T, Treiber N, Meister G. Regulation of microRNA biogenesis and its crosstalk with other cellular pathways. Nat Rev Mol Cell Biol.2019;20:5-20.

11. Song $H$, Nan $Y$, Wang $X$, et al. MicroRNA613 inhibits proliferation and invasion of renal cell carcinoma cells through targeting FZD7. Mol Med Rep. 2017;16:4279-4286.

12. Yu X, Wang W. Tumor suppressor microRNA613 inhibits glioma cell proliferation, invasion and angiogenesis by targeting vascular endothelial growth factor A. Mol Med Rep. 2017;16:6729-6735.

13. Liu C,Jiang Y,Han B,miR-613 Suppresses Chemoresistance and Stemness in Triple-Negative Breast Cancer by Targeting FAM83A.Cancer Manag Res.2020;12: 12623-12633.

14. Ji H,Hu NJ.MiR-613 blocked the progression of cervical cancer by targeting LETM1. Eur Rev Med Pharmacol Sci.2020; 24: 6576-6582.

15. Sargent KM, Clopton DT, Lu N,et al. VEGFA splicing: divergent isoforms regulate spermatogonial stem cell maintenance. Cell Tissue Res. 2016;3631:31-45

16. Reinmuth N, Parikh AA, Ahmad SA, et al. Biology of angiogenesis in tumors of the gastrointestinal tract. Microsc Res Tech. 2003;60:199-207.

17. Itakura J, Ishiwata T, Shen B, et al. Concomitant over-expression of vascular endothelial growth factor and its receptors in pancreatic cancer. Int J Cancer. 2000;85:27-34.

18. Seo Y, Baba H, Fukuda T, et al. High expression of vascular endothelial growth factor is associated with liver metastasis and a poor prognosis for patients with ductal pancreatic adenocarcinoma. Cancer. 2000;88:2239-45.

19. Zhong Z,Huang M,Lv M, et al. Circular RNA MYLK as a competing endogenous RNA promotes bladder cancer progression through modulating VEGFA/VEGFR2 signaling pathway .Cancer Lett. 2017; 403: 305-317.

20. Zhang Q,Lu S,Li T, et al. ACE2 inhibits breast cancer angiogenesis via suppressing the VEGFa/VEGFR2/ERK pathway. J Exp Clin Cancer Res. 2019; 38: 173.

21. Wang Y, Zhang F, Wang J, et al. IncRNA LOC100132354 promotes angiogenesis through VEGFA/VEGFR2 signaling pathway in lung adenocarcinoma.Cancer Manag Res 2018;10:4257-4266

22. Noh JH, Kim KM, McClusky WG, Abdelmohsen K, Gorospe M. Cytoplasmic functions of long noncoding RNAs. Wiley Interdiscip Rev RNA. 2018; 9: e1471.

23. Salzman J, Gawad C, Wang PL, et al. Circular RNAs are the predominant transcript isoform from hundreds of human genes in diverse cell types. PLoS One. 2012; 7: e30733.

24. Liu J, Liu T, Wang X, et al. Circles reshaping the RNA world: from waste to treasure. Mol Cancer. 2017; 16: 58 .

25. Rong Zeyin,Xu Jin,Shi Si ,et al. Circular RNA in pancreatic cancer: a novel avenue for the roles of diagnosis and treatment . Theranostics. 2021, 11: 2755-2769. 
26. Huang L,Han J,Yu H ,et al. CircRNA_000864 Upregulates B-cell Translocation Gene 2 Expression and Represses Migration and Invasion in Pancreatic Cancer Cells by Binding to miR-361-3p.Front Oncol. 2020, 10: 547942.

27. Ye Z,Zhu Z,Xie J, et al. Hsa_circ_0000069 Knockdown Inhibits Tumorigenesis and Exosomes with Downregulated hsa_circ_0000069 Suppress Malignant Transformation via Inhibition of STIL in Pancreatic Cancer.Int J Nanomedicine. 2020, 15: 9859-9873.

28. Zhang X,Tan P,Zhuang Y, et al. hsa_circRNA_001587 upregulates SLC4A4 expression to inhibit migration, invasion, and angiogenesis of pancreatic cancer cells via binding to microRNA-223.Am J Physiol Gastrointest Liver Physiol. 2020, 319: G703-G717.

29. Kong Y,Li Y,Luo Y, et al. circNFIB1 inhibits lymphangiogenesis and lymphatic metastasis via the miR486-5p/PIK3R1/VEGF-C axis in pancreatic cancer.Mol Cancer. 2020, 19: 82.

30. Claesson-Welsh L,Welsh M,VEGFA and tumour angiogenesis .J Intern Med, 2013, 273: 114-

31. Mastrella G, Hou M, Li M,et al.Targeting APLN/APLNR Improves Antiangiogenic Efficiency and Blunts Proinvasive Side Effects of VEGFA/VEGFR2 Blockade in Glioblastoma.Cancer Res 2019;79(9):

32. Fontanella C, Ongaro E, Bolzonello S,et al. Clinical advances in the development of novel VEGFR2 inhibitors.Ann Transl Med 2014; 2 (12): 123.

\section{Figures}


A
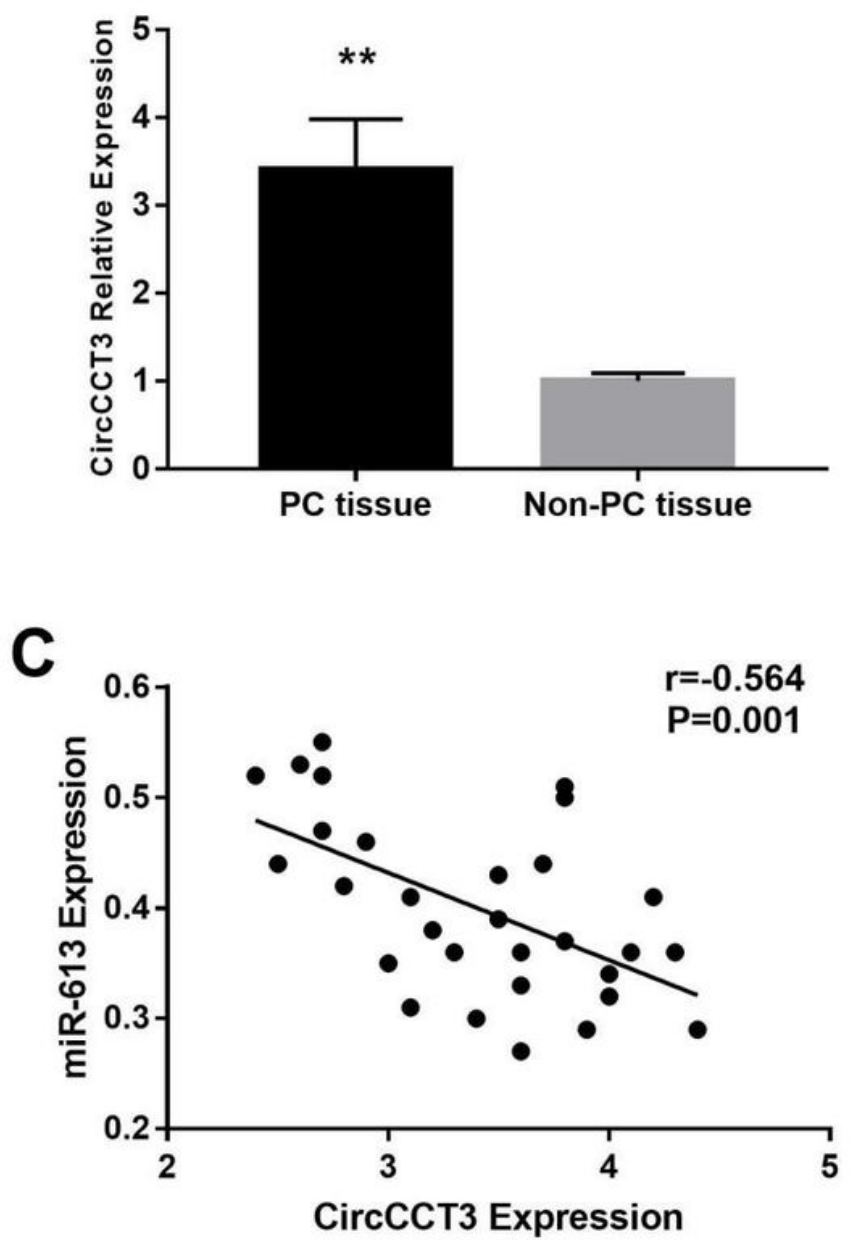

B

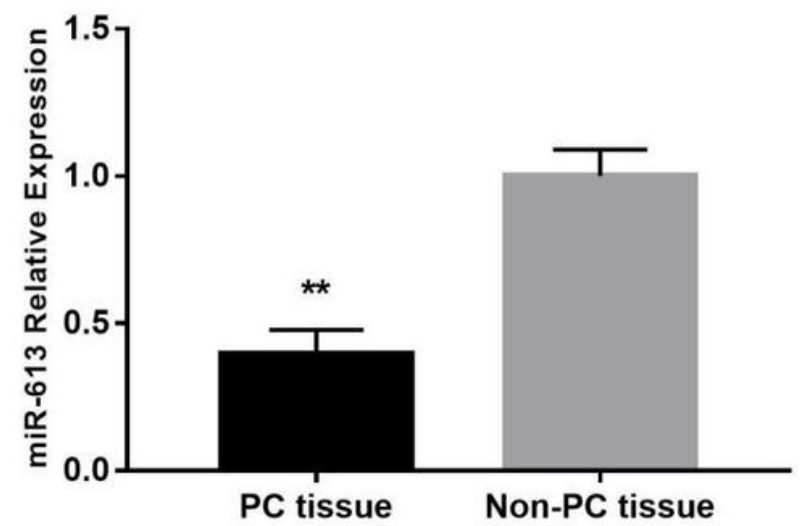

D

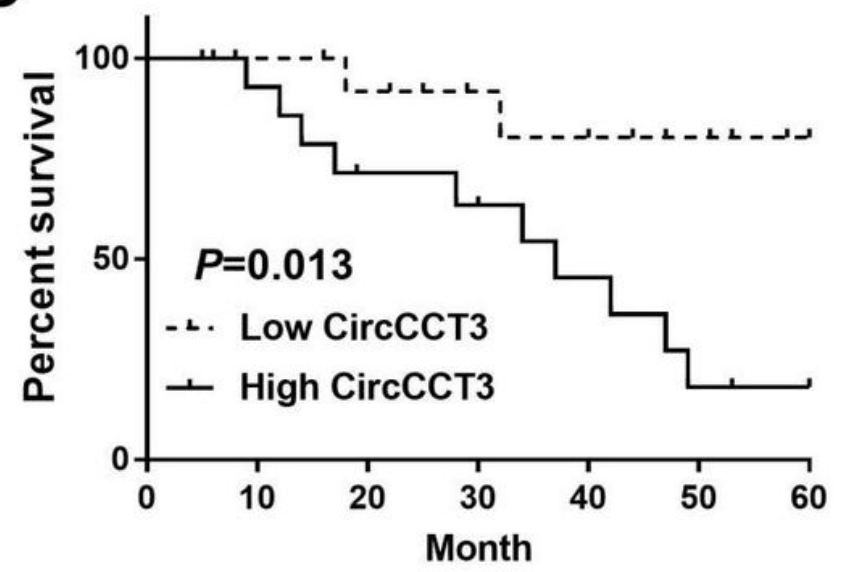

Figure 1

A. Expression levels of CircCCT3 in PC samples and adjacent non-PC tissue. B. Expression levels of miR613 in PC samples and adjacent non-PC tissue C. Correlation of CircCCT3 expression with miR-613 expression in PC D. Pancreatic carcinoma patients with high CircCCT3 expression $(n=15)$ exhibited significantly poorer overall survival rate than those patients with low CircCCT3 expression $(n=15)$ as defined by log-rank test $(P=0.013)$. 
A

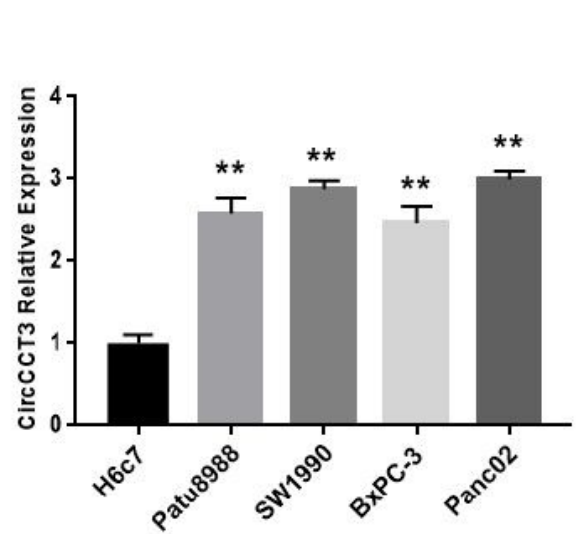

D

\section{OE-CircCCT3}

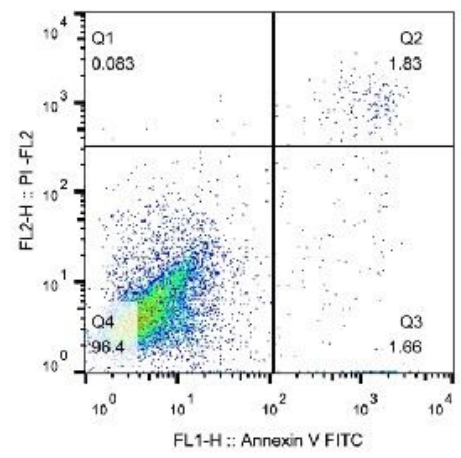

B

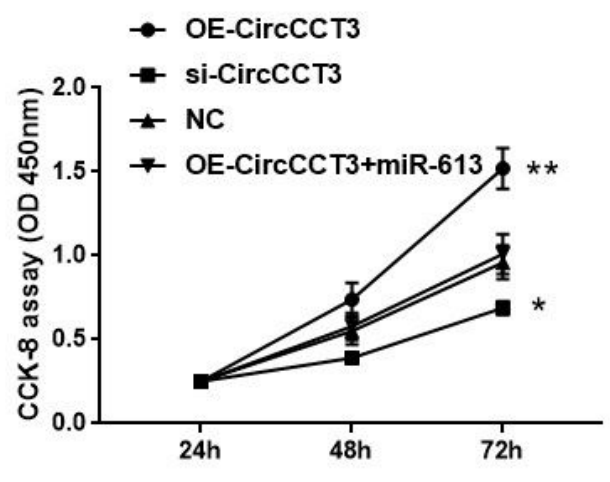

si-CircCCT3

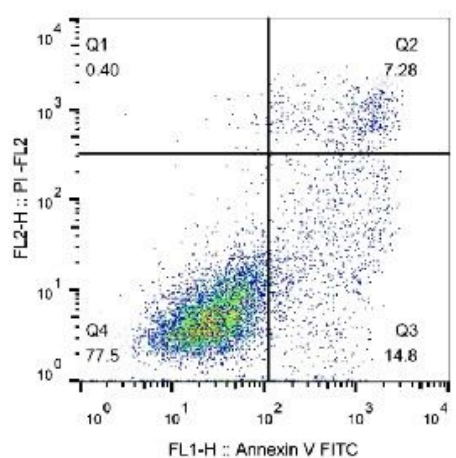

C

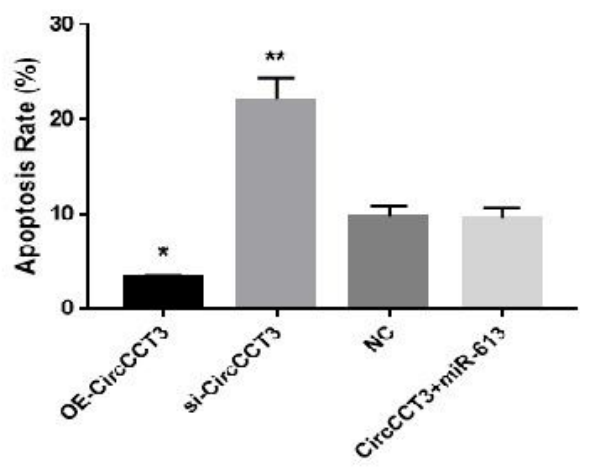

OE-CircCCT3

miR-613 mimic

\section{Figure 2}
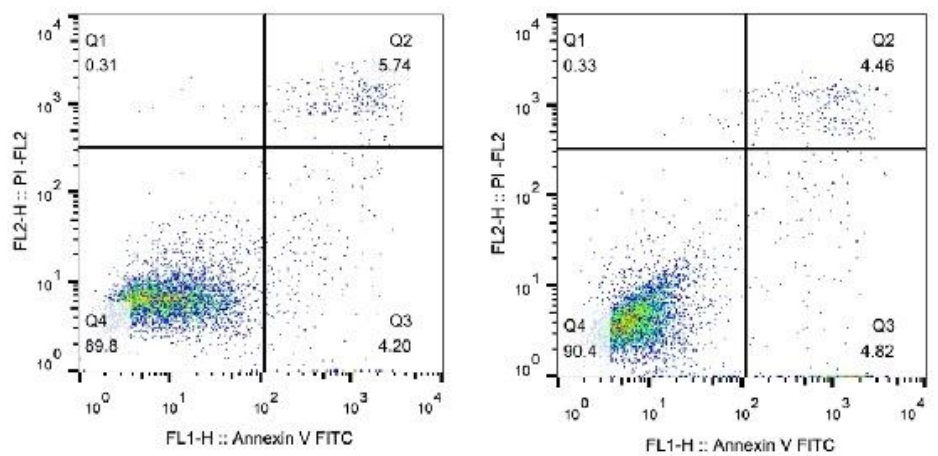

A. Comparison of CircCCT3 levels between normal cell line $\mathrm{H} 6 \mathrm{c} 7$ and different tumor cell lines B. Proliferation rate following OE-CircCCT3 or si-CircCCT3 transfection C. Comparison of apoptosis rate following OE-CircCCT3 or si-CircCCT3 transfection D. Represetative figures of cell apoptosis following OECircCCT3 or si-CircCCT3 transfection 
A
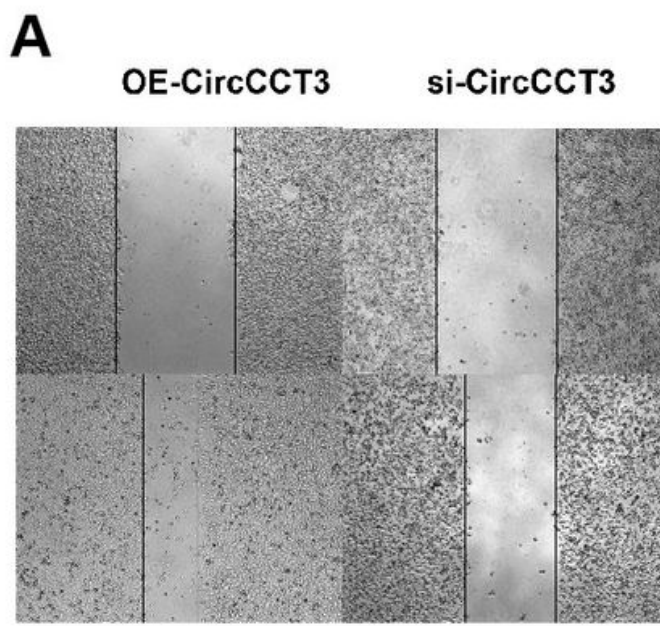

NC

OE-CircCCT3

B

miR-613 mimic

C OE-CircCCT3 si-CircCCT3

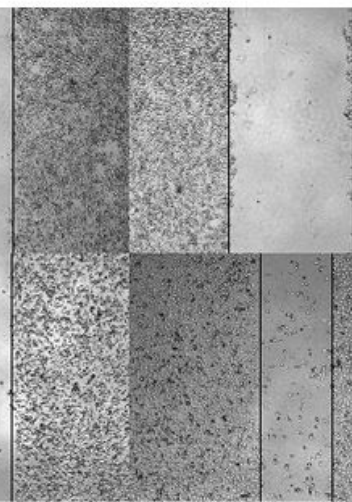

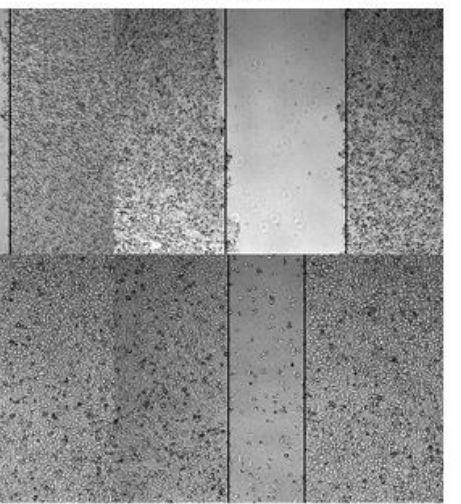

OE-CircCCT3 miR-613 mimic

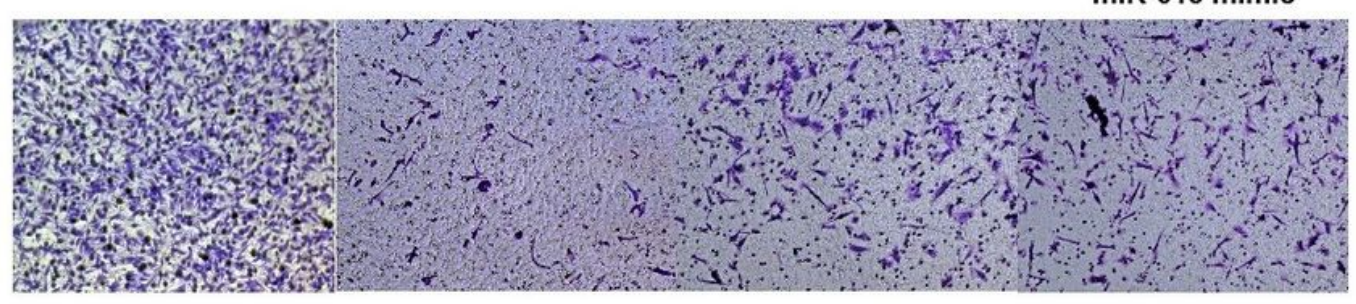

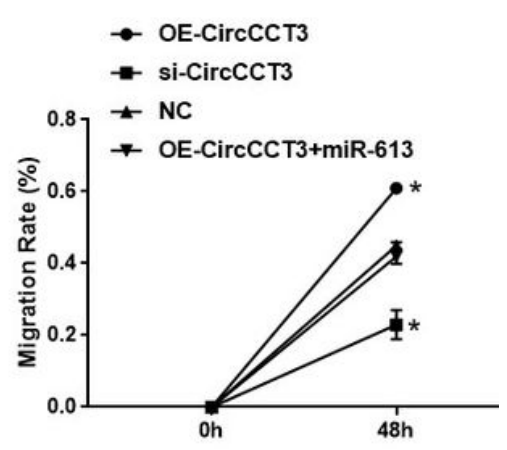

D

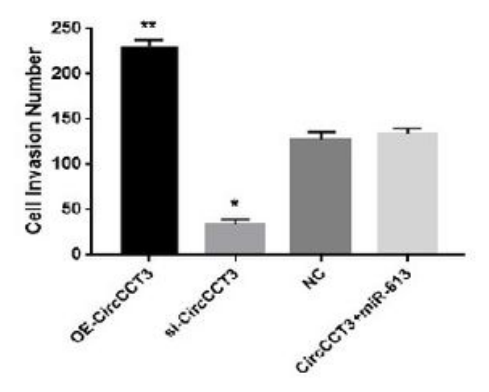

Figure 3

A. B. Wound healing assay for Panc02 cells migration after $48 \mathrm{~h}$ following OE-CirCCT3 or OE- CirCCT3 $\left({ }^{\star} \mathrm{P}<0.05,{ }^{\star \star} \mathrm{P}<0.01\right)$ C . D. Transwell Invasion assay for Panc02 cells invasion after $48 \mathrm{~h}$ using in $\mathrm{Panc02}$ cells following OE-CirCCT3 or OE- CirCCT3 ( ${ }^{*}<0.05$, ${ }^{\star *} \mathrm{P}<0.01$ vs NC) 


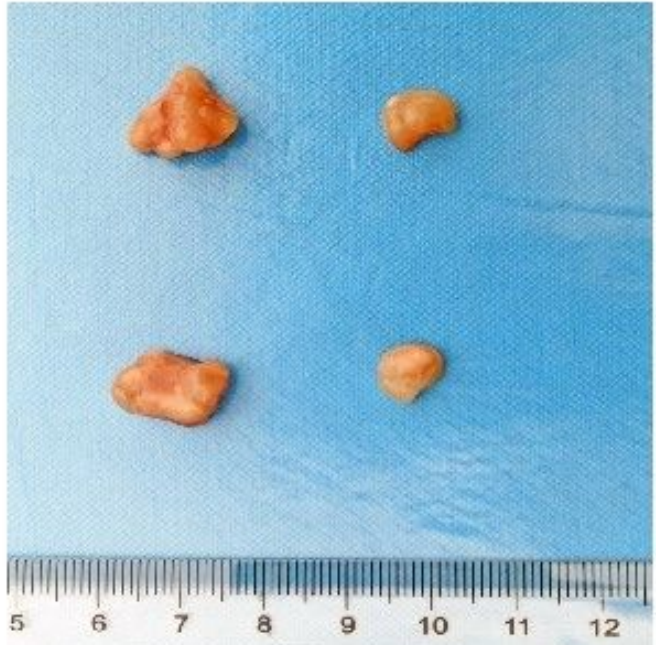

C

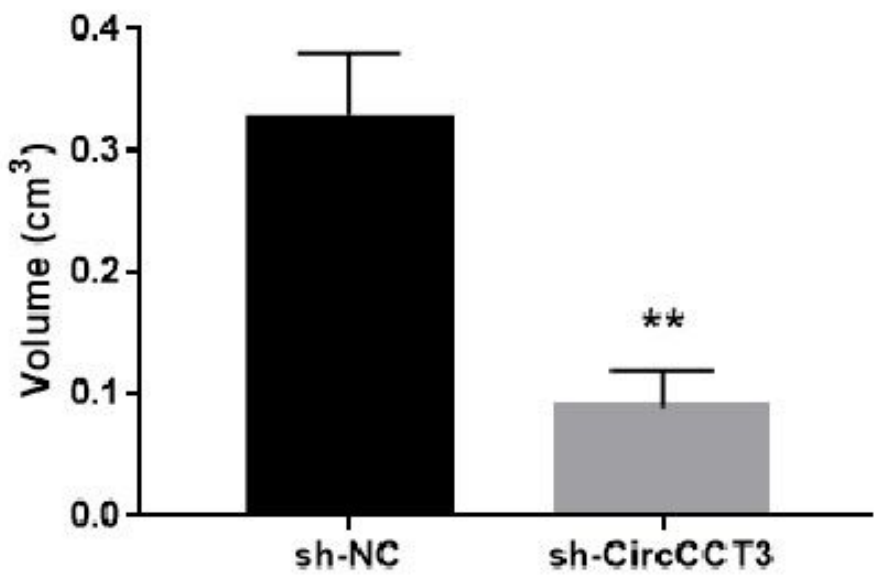

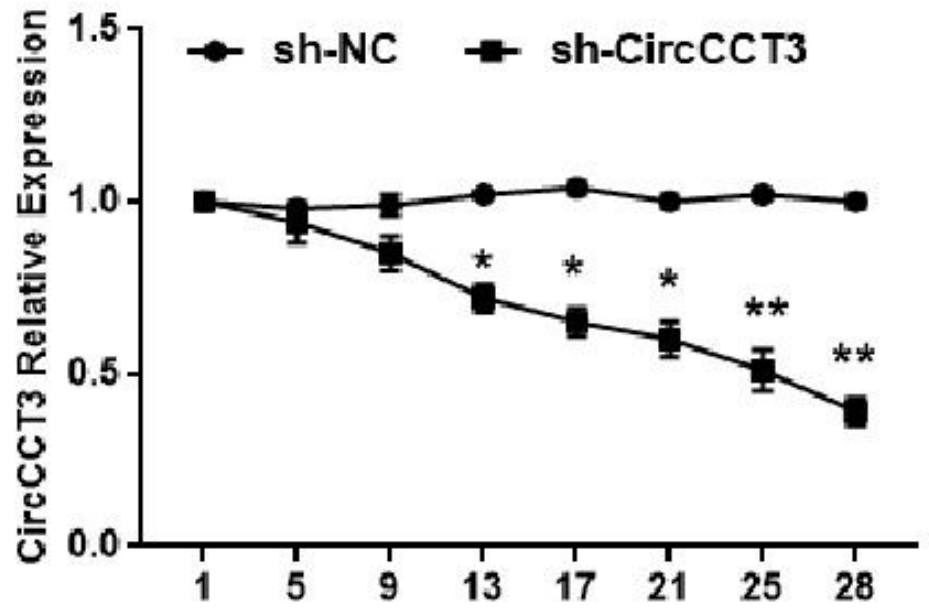

D

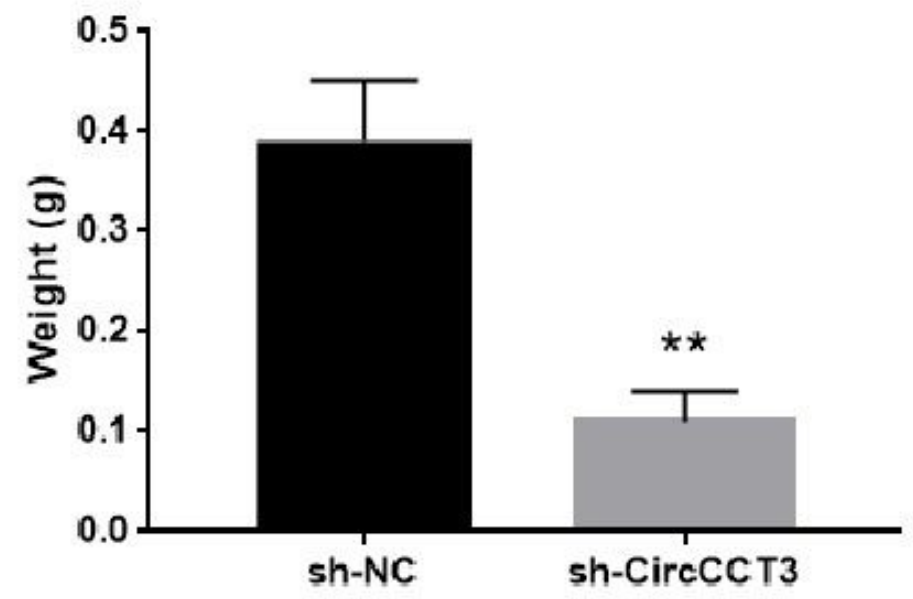

Figure 4

sh-CirCCT3 suppressed tumor growth of PC. A Representative photographs of PC tumor tissue in shCirCCT3 and sh-CircNC groups. B. Expressions of CircCCT3 during the tumor growth between si-CirCCT3 and si-CircNC groups. C. Comparison of tumor volume between sh-CircCCT3 group and sh-CircNC group at day 24 ( $* * P<0.01)(n=6$ for each group) D. Comparison of tumor weight between sh-CircCCT3 group and sh-CircNC group tumor volume at day $24\left({ }^{*} \mathrm{P}<0.01\right)(\mathrm{n}=6$ for each group) 
miR-613: 3' CCGUUUCUUCCUUGUAAGGA 5' CircCCT3 : 5' AggCCCUAGagguCAUUUCCU 3'

MutCircCCT3: 5' AGGCCCUAGAGGUUGCCAGC 3'

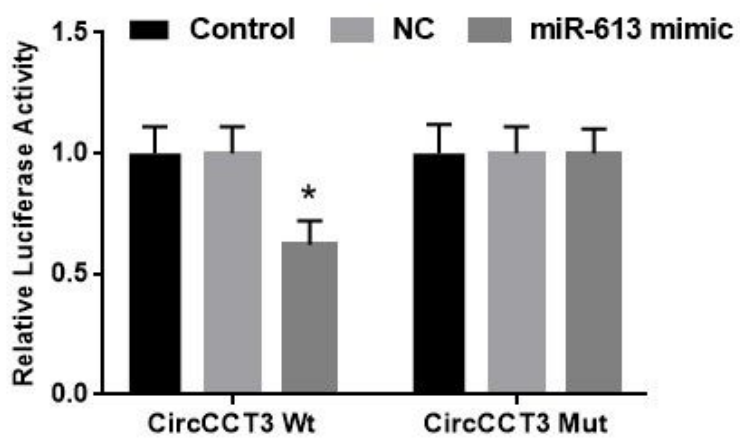

C

VEGFA 3'-UTR: 5' CUGGCUCCCCAGCACACAUUCCU 3'

MUT VEGFA 3'-UTR 5' CUGGCUCCCCAGCACCACGGAAG 3'
D

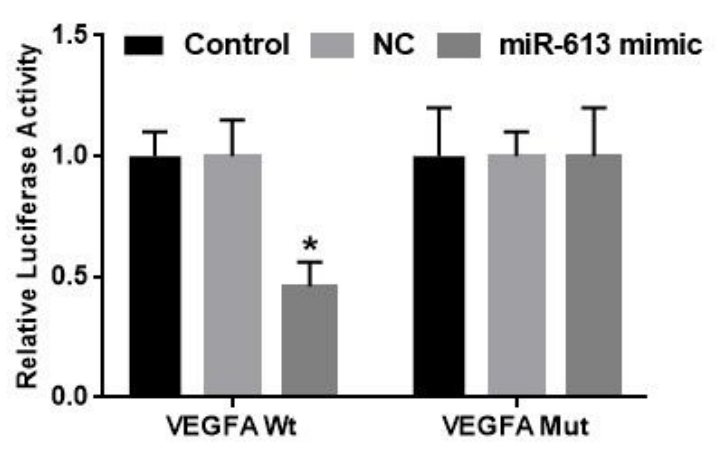

Figure 5

A. Bioinformatics analysis of matching sequence of miR-613 within CircCCT3. MUT CircCCT3 is the mutation of the match sequence of of CircCCT3 with miR-613. B Luciferase reporter assay revealed that miR-613 binds to the CircCCT3, not MUT CircCCT3. C. Bioinformatics analysis of matching sequence of miR-613 within 3'-UTR of VEGFA. MUT VEGFA 3'-UTR is the mutation of the match sequence of 3'-UTR of VEGFA with miR-613. D. Luciferase reporter assay revealed that miR-613 binds to the 3'-UTR of WT VEGFA, not MUT VEGFA. Relative luciferase activity was quantified and the data were demonstrated as mean $\pm S D$. ${ }^{*} P<0.05$ vs. respective NC groups; NC, negative control; $W T$, wild-type; MUT, mutant. 

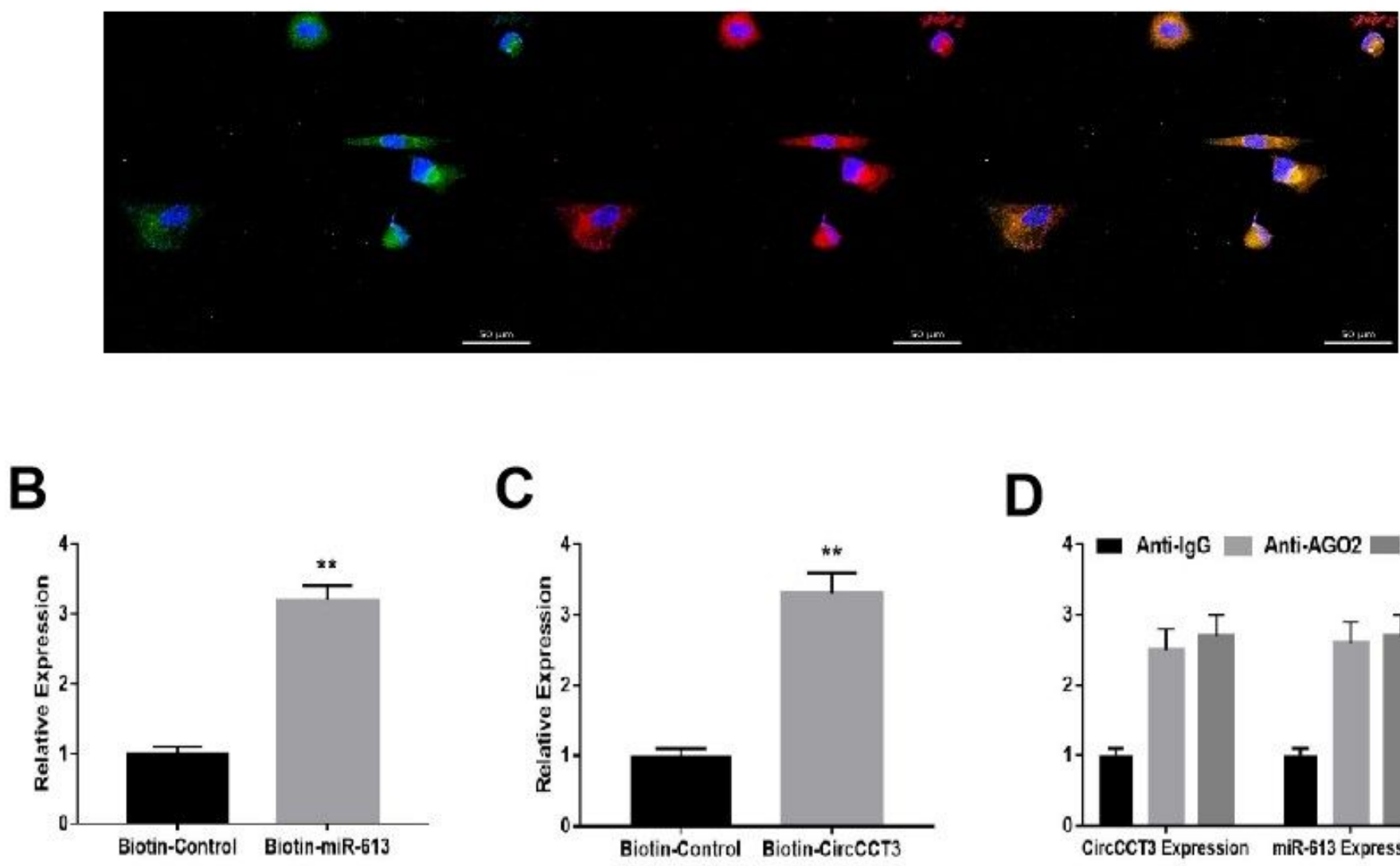

\section{D}

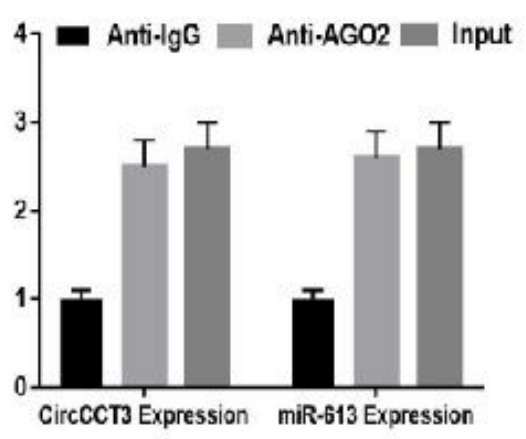

Figure 6

A. CircCCT3 and miR-613 were colocalized in Panc02 cells by FISH using confocal microscope. CircCCT3 was stained red, miR-613 was stained green, nuclei were stained blue (DAPI) and overlapped expression was mixed (Scale bar, $20 \mu \mathrm{m}$ ).B. The biotin-coupled probe pull-down assay was performed and the results showed miR-613 was detected in the CircCCT3 pulled-down pellet compared with the control group. C. CircCCT3 was detected in the biotin-miR-613 vector compared with the control group D. Relative CircCCT3 and miR-613 expressions presented as fold enrichment in Ago2 relative to normal IgG immunoprecipitates. RIP assays disclosed that CircCCT3 and miR-613 expressions were substantially enriched by Ago2 antibody compared with control IgG antibody. ( $P<0.01 v s$ control) 
A
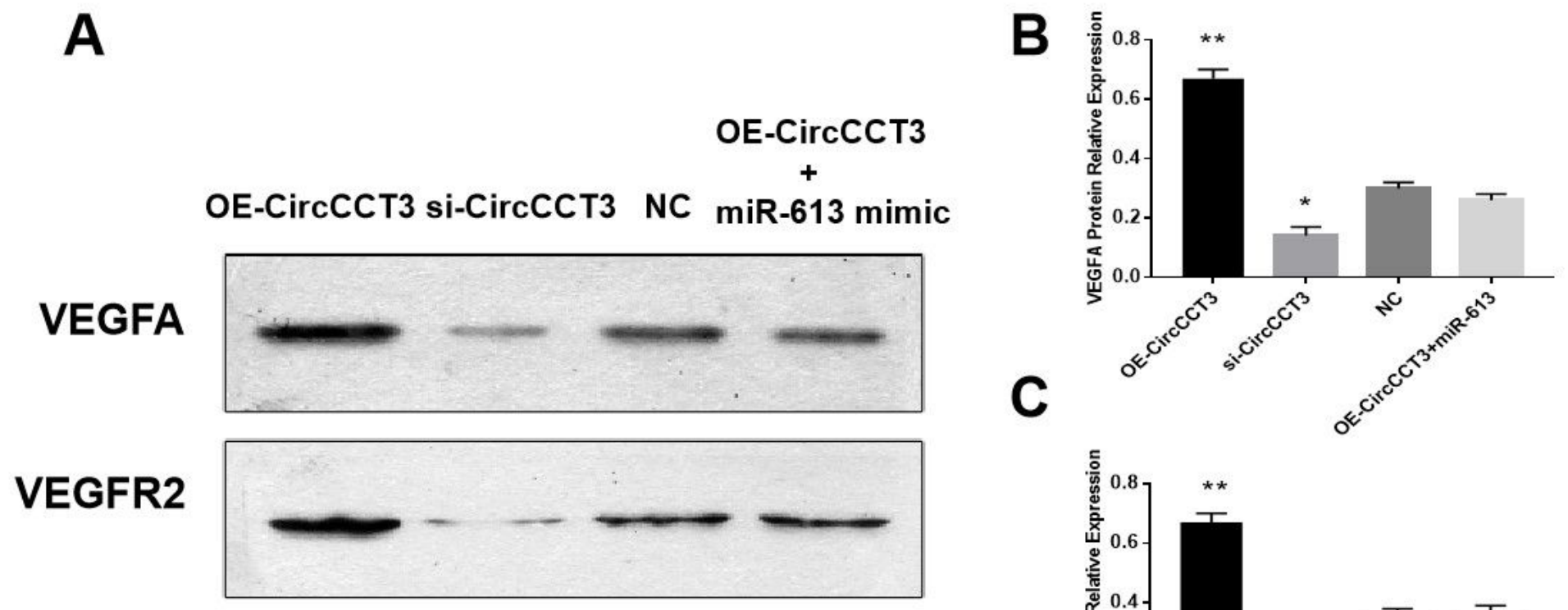

\section{GAPDH}
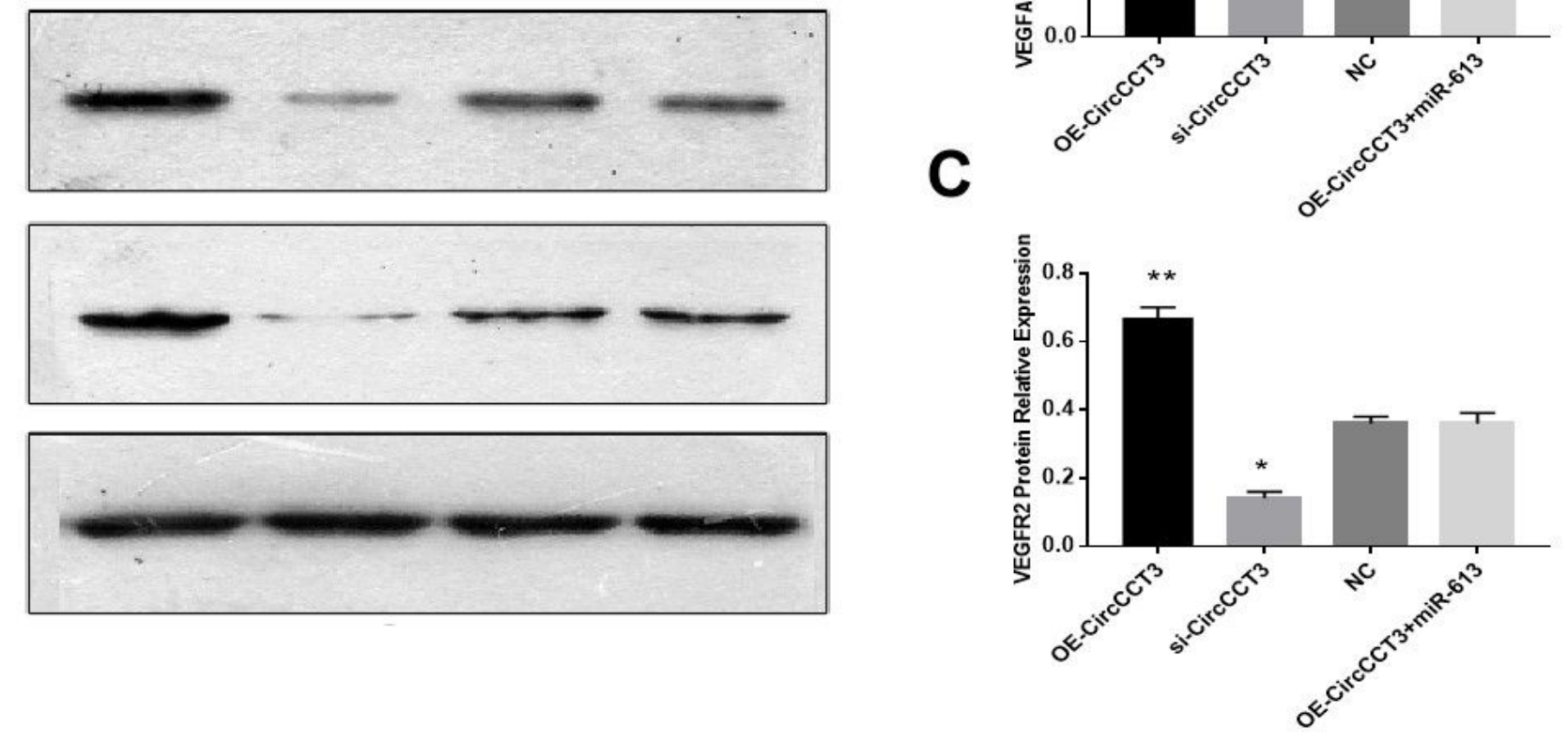

Figure 7

VEFGA and VEGFR2 protein expressions detected through western blot analysis. A. Representative bands of VEGFA and VEGFR2 B. Relative expressions of VEGFA proteins following OE-CircCCT3 or si-CircCCT3 transfection C. Relative expressions of VEGFR2 proteins following OE-CircCCT3 or si-CircCCT3 transfection 